\title{
A real-time machine-vision system for monitoring the textile raising process
}

\author{
Monica Carfagni, Rocco Furferi, Lapo Governi
}

\begin{abstract}
This work provides an automatic and non-intrusive tool to objectively monitoring the raising process by measuring the height and the density of the fibres emerging from a raised cloth (pile). These parameters are assessed by a numerical procedure, which elaborates the images provided by an appositely developed machine-vision system. The proposed approach allows the investigation and the control of the raising process and has been validated by experimental measurements performed on a set of specimens (cloths) with several raising degrees. The comparison between the results obtained by the proposed procedure and the ones coming from a widely accepted textile-measuring device (fabric assurance by simple testing, FAST) is also provided.
\end{abstract}

\# 2005 Elsevier B.V. All rights reserved. 